\title{
Multi-dimensional SAR tomography for monitoring the deformation of newly built concrete buildings
}

\author{
Peifeng Ma ${ }^{a}$, Hui Lin ${ }^{\mathrm{a}, *}$, Hengxing Lan ${ }^{\mathrm{b}}$, Fulong Chen ${ }^{\mathrm{c}}$ \\ a Institute of Space and Earth Information Science, Department of Geography and Resource Management and Shenzhen Research Institute, The Chinese University of Hong Kong, \\ Shatin, N.T., Hong Kong \\ ${ }^{\mathrm{b}}$ State Key Laboratory of Resources and Environmental Information System (LREIS), Institute of Geographic Sciences and Natural Resources Research, Chinese Academy of \\ Sciences, Beijing 100101, China \\ ${ }^{\mathrm{c}}$ Key Laboratory of Digital Earth Science, Institute of Remote Sensing and Digital Earth, Chinese Academy of Sciences, Beijing 100094, China
}

\section{A R T I C L E I N F O}

\section{Article history:}

Received 22 December 2014

Received in revised form 8 April 2015

Accepted 20 April 2015

Available online 8 June 2015

\section{Keywords:}

Multi-dimensional SAR tomography

Newly built concrete buildings

Thermal expansion

Creep and shrinkage

TerraSAR-X

\begin{abstract}
A B S T R A C T
Deformation often occurs in buildings at early ages, and the constant inspection of deformation is of significant importance to discover possible cracking and avoid wall failure. This paper exploits the multi-dimensional SAR tomography technique to monitor the deformation performances of two newly built buildings (B1 and B2) with a special focus on the effects of concrete creep and shrinkage. To separate the nonlinear thermal expansion from total deformations, the extended 4-D SAR technique is exploited. The thermal map estimated from 44 TerraSAR-X images demonstrates that the derived thermal amplitude is highly related to the building height due to the upward accumulative effect of thermal expansion. The linear deformation velocity map reveals that $\mathrm{B} 1$ is subject to settlement during the construction period, in addition, the creep and shrinkage of B1 lead to wall shortening that is a height-dependent movement in the downward direction, and the asymmetrical creep of B2 triggers wall deflection that is a height-dependent movement in the deflection direction. It is also validated that the extended 4-D SAR can rectify the bias of estimated wall shortening and wall deflection by 4-D SAR.

(c) 2015 International Society for Photogrammetry and Remote Sensing, Inc. (ISPRS). Published by Elsevier
\end{abstract} B.V. All rights reserved.

\section{Introduction}

\subsection{Multi-dimensional SAR tomography}

Multi-dimensional synthetic aperture radar (SAR) tomography (MD-TomoSAR) (De Maio et al., 2009; Fornaro et al., 2009; Lombardini, 2005; Reale et al., 2011; Zhu and Bamler, 2011), which originates from three-dimensional SAR tomography (3-D SAR) (Budillon et al., 2011; Fornaro et al., 2003; Ma et al., 2014; Reigber and Moreira, 2000; Zhu and Bamler, 2010), is developed for measuring the slow deformation of single and overlaid multiple persistent scatterers. This technology can be seen as an extension of the conventional persistent scatterer interferometry (PSI) technique (Ferretti et al., 2000) in the context of SAR tomography. Conventional PSI can only detect single scatterers, whereas in addition to single scatterers, MD-TomoSAR can identify multiple scatterers that often arise in built-up regions with steep surface topography or densely spaced infrastructures (Fornaro et al.,

\footnotetext{
* Corresponding author. Tel.: +852 39436010.

E-mail address: huilin@cuhk.edu.hk (H. Lin).
}

2009). The joint detection of single and multiple scatterers increases the density of deformation measurements. Currently, MD-TomoSAR primarily consists of differential SAR tomography (4-D SAR) (Fornaro et al., 2009; Lombardini, 2005) and the extended 4-D SAR (Reale et al., 2011; Zhu and Bamler, 2011). They differ from each other in terms of the exploited deformation model. 4-D SAR uses a linear deformation model to approximate the deformation phase and aims to estimate the height and linear deformation velocity by reconstructing tomography in the spacetime domain (Lombardini, 2005). Compared with conventional PSI, apart from resolving multiple scatterers, 4-D SAR exploits phase as well as amplitude information rather than only phase information for estimation. This change leads to a better detection probability according to constant false alarm rate analysis (De Maio et al., 2009). As the shorter wavelength X-band SAR sensors (typically, TerraSAR-X and Cosmo-Skymed) can measure subtle movements, such as thermal expansion, more sensitively (Crosetto et al., 2015), the linear deformation model that is used in 4-D SAR becomes insufficient to approximate the deformation phase, especially when monitoring concrete structures that are sensitive to thermal expansion, leading to so-called underfitting 
effects (Colesanti et al., 2003). The underfitting effects have a considerably negative impact on the accuracy of the estimated linear velocity in the sense that the linear velocity may include partial nonlinear thermal expansion. In addition, underfitting effects may make some structures undetectable due to a lower goodness of fit (Reale et al., 2013), which will cause loss of significant structural details in the monitoring. To increase the goodness of fit, 4-D SAR has been further extended to monitor nonlinear thermal expansion by recovering tomography in the space-time-tempera ture domain. In (Zhu and Bamler, 2011) the extended 4-D SAR is introduced for the first time, and it is further analyzed in (Fornaro et al., 2013; Reale et al., 2013). The extended 4-D SAR is an extension of the extended PSI (Monserrat et al., 2011) in the context of SAR tomography. The extended 4-D SAR adds a nonlinear temperature model to the conventional linear deformation model when expressing the deformation phase (Fornaro et al., 2013; Reale et al., 2013). As a consequence, in addition to height and linear deformation velocity, we can obtain a new geophysical parameter using the extended 4-D SAR, namely thermal amplitude. Thermal amplitude, which indicates the movement level of structural components associated with changes in temperature, depends primarily on the properties of construction materials and the geometric shape of structures (Monserrat et al., 2011). An alternative for the compensation of thermal expansion is to use a seasonal sinusoidal function (Gernhardt and Bamler, 2012) to approximate the thermal expansion based on the fact that thermal expansion is normally periodic with seasonal temperature variations, which is feasible when the temperature is unknown. Compared with sinusoidal functions, the temperature model is related to physical reality more directly and is thus more accurate (Crosetto et al., 2015). A critical error source of the extended 4-D SAR is the possible correlation between the distributions of time and temperature, which may degrade the accuracy of the estimated linear deformation velocity and thermal amplitude (Reale et al., 2013). To overcome this problem, the number of available images should be large enough (the larger, the better) and the total temporal span should be long enough (the longer, the better) (Crosetto et al., 2015; Reale et al., 2013).

\subsection{Slow deformation of newly built concrete buildings}

Deformation often occurs in buildings at early ages, and the constant inspection of deformation is of significant importance to discover possible cracking and avoid wall failure. Currently, most buildings are built of concrete (e.g., plain concrete and reinforced concrete) because of concrete's low cost, durability, and high compressive strength. The slow deformation of newly built concrete buildings is primarily composed of thermal expansion, possible settlement, creep and shrinkage, of which creep and shrinkage only occur in concrete structures. Although buildings can also deform under other driving forces, such as wind and accidental load, SAR is not applicable for quantifying these instant movements and hence we will not consider these forces in our study.

Thermal expansion, which is an intrinsic characteristic of construction materials, means that structural components will expand or contract periodically when subjected to changes in temperature. The expansion level of structures is linearly related to the thermal expansion coefficient of the construction materials. Thermal expansion may trigger structural cracking in structures if not properly managed. In practice, to avoid possible thermal cracking, some expansion gaps or joints should be designed in response to changes in temperature. Newly built buildings, especially high-rise buildings, may be subject to settlement, which is normally caused by the high load or consolidation of foundation. Generally, a total settlement rate up to $4 \mathrm{~mm} / \mathrm{y}$ is allowable in engineering (Van Waning, 2014). If the settlements do not cause security problems, larger settlements may be still acceptable. In addition to thermal expansion and possible settlement, creep and shrinkage are another two long term movements in concrete structures that may influence structural health. When a load is applied on a concrete structure, the structure first shows an instantaneous deformation which is then followed by slow further increase of deformation. This slow increase of deformation is called creep (Bazant and Wittmann, 1982). The rate of creep depends primarily on the material properties, structural load, exposure time, and exposure temperature. Concrete specimens slowly deform over time even in the absence of applied load. These deformations are called shrinkage when the temperature is constant (Bazant and Wittmann, 1982). Concrete shrinkage is a volume contraction due to a loss of moisture, and is primarily affected by the humidity of the surrounding air. Normally, the rates of creep and shrinkage are largest at the outset and gradually decline as the load is relaxed and dehydration proceeds. Newly built concrete buildings contract in volume under the forces of creep and shrinkage and reach a steady state as time approaches infinity (Gilbert, 2001).

The deformation monitoring of buildings using interferometric SAR (InSAR) methods has been performed in some studies, e.g., (Crosetto et al., 2015; Gernhardt et al., 2010; Gernhardt and Bamler, 2012; Reale et al., 2013; Van Waning, 2014). The primary purpose of this paper is to explore the possibility and potential of MD-TomoSAR for the deformation monitoring of newly built concrete buildings with a special focus on the effects of concrete shrinkage and creep. In Section 2, we describe the data set and the method that we used in our experiments. Two buildings are newly built of concrete in our study site. To remove thermal expansion, the extended 4-D SAR is exploited for deformation monitoring. In Section 3, the derived height, thermal amplitude, and linear deformation velocity of the study site are presented. The deformation performances of the two newly built buildings, including thermal expansion, settlement, concrete creep and shrinkage are demonstrated. In particular, wall shortening and wall deflection incurred by concrete creep and shrinkage are analyzed in detail. For comparison, we also present the estimates by 4-D SAR. Finally, we summarize several conclusions in Section 4.

\section{Data set and methodology}

The SAR data that were used in our experiments are TerraSAR-X images. The sensor looks down on the study site from the west at an incidence angle of 37.4 degrees. The images are collected from November 2008 to December 2010 in the ascending mode. The data comprise 41 TerraSAR-X and 3 TanDEM-X strip-map mode images with a range spacing resolution of approximately $0.9 \mathrm{~m}$ and an azimuth spacing resolution of approximately $2 \mathrm{~m}$. The polarization mode is VV channel. The distributions of the temporal baseline and spatial perpendicular baseline are depicted in Fig. 1 (top) with the image acquired in November 2009 as the master image. The mean SAR amplitude image of the study site with a coverage of 1900 pixels $\times 1200$ pixels is shown in Fig. 1 (bottom). This district includes many high-rise buildings and transportation facilities. Two buildings are newly built in this area: B1 in the red rectangle and B2 in the yellow rectangle. The building B1 was still under construction at the beginning time of the acquisitions and was completed in the first half of 2010 (the red triangle in Fig. 1 (top)), the final height of B1 is close to $500 \mathrm{~m}$. The building B2 was completed in the first half of 2008 (the yellow triangle in Fig. 1 (top)), and the height is approximately $250 \mathrm{~m}$.

As described above, newly built concrete buildings are subject to thermal expansion, possible settlement, creep and shrinkage. To separate thermal expansion from possible settlement, creep, and shrinkage, the extended 4-D SAR technique is exploited in 

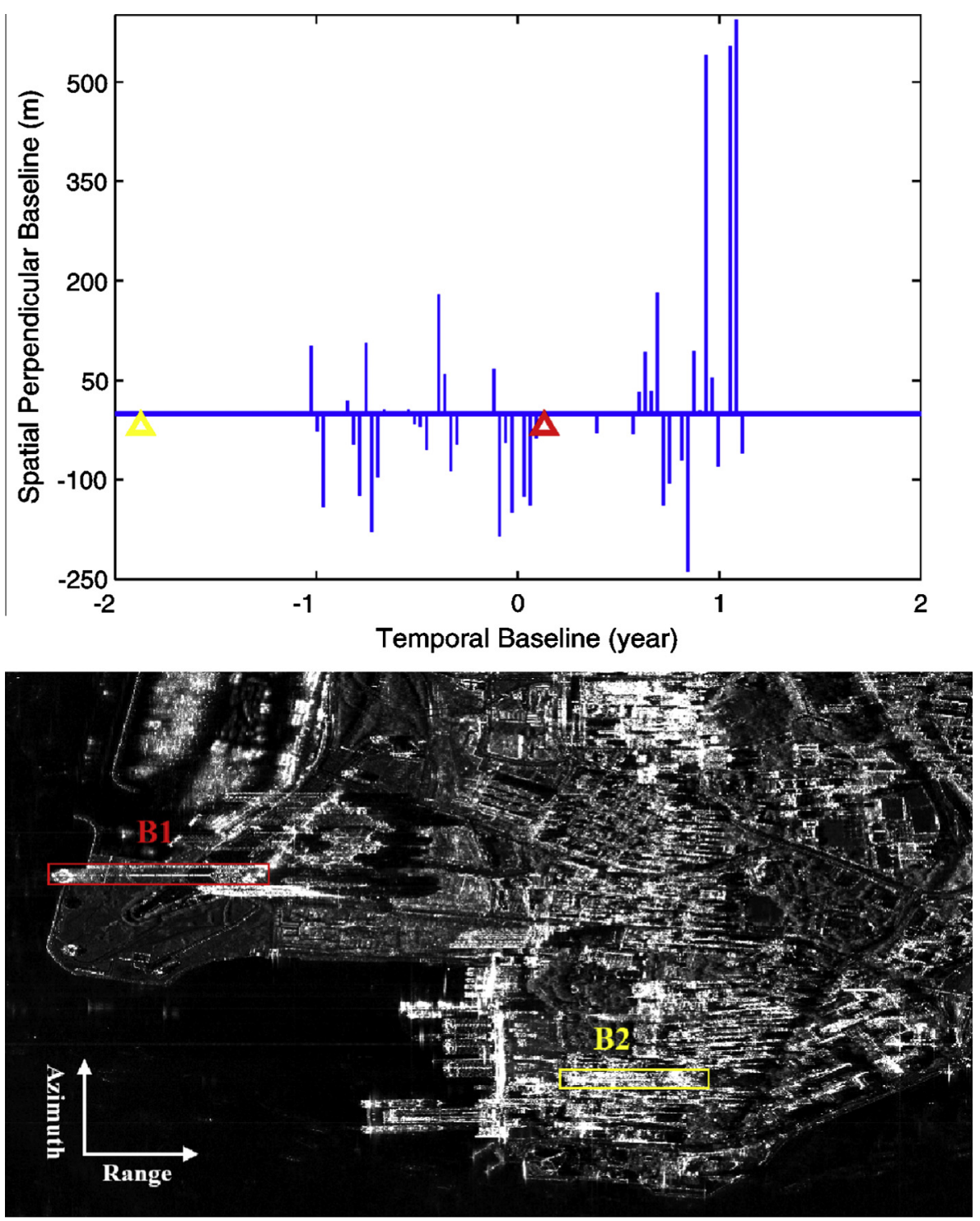

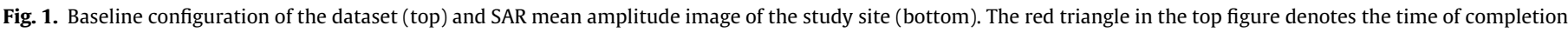

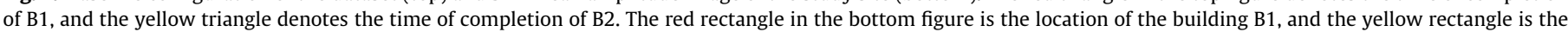
location of the building B2. (For interpretation of the references to colour in this figure legend, the reader is referred to the web version of this article.)

our study. Amplitude and phase calibrations are carried out before tomographic inversion. The relative amplitude calibration can be simply completed by the following equation:

$x_{c}=x / X$

where $x_{c}$ is the calibrated amplitude of one pixel, $x$ is the original amplitude, and $X$ stands for the mean amplitude value of each image. The phase calibration includes differential interferometric processing and removal of atmospheric disturbance. Differential interferometry is conducted by Gamma software. Then we use a method based on the construction of network in PSI (Colesanti et al., 2003; Ferretti et al., 2000) to remove atmospheric disturbance. Note that the extended 4-D SAR phase model is employed when extracting atmospheric disturbance to avoid filtering thermal expansion. Assuming a spatially separated array consisting of $N$ repeat-pass sensors illuminating the target, the general signal model of SAR tomography can be written as follows (Stoica and Moses, 2005):

$\boldsymbol{y}=\boldsymbol{A} \gamma+\boldsymbol{e}$ where $\boldsymbol{y}$ is the vector collecting the received signals after amplitude and phase calibrations, $\boldsymbol{A}$ is the sensing matrix containing steering vectors $\boldsymbol{a}$ as columns, $\gamma$ is the reflectivity profile to be solved, i.e., the tomography, and $\boldsymbol{e}$ is the vector containing noise. After a discretization over a set of $M_{s}$ points along the slant elevation direction $s$, a discretization over a set of $M_{v}$ points along the linear deformation velocity direction $v$, and a discretization over a set of $M_{k}$ points along the thermal amplitude direction $k$, we can obtain (Zhu and Bamler, 2011):

$\boldsymbol{A}=\left[\boldsymbol{a}\left(s_{1}, v_{1}, k_{1}\right), \ldots, \boldsymbol{a}\left(s_{M_{s}}, v_{M_{v}}, k_{M_{k}}\right)\right]$

and

$\boldsymbol{a}(s, v, k)=\left[\exp \left(j 2 \pi\left(\xi_{1} s+\eta_{1} v+\zeta_{1} k\right)\right), \ldots, \exp \left(j 2 \pi\left(\xi_{N} s+\eta_{N} v+\zeta_{N} k\right)\right)\right]^{T}$

where $\xi_{i}=2 b_{i} / \lambda r_{0}, i=1, \ldots, N\left(b_{i}\right.$ is the spatial perpendicular baseline with respect to the master image, $\lambda$ is the wavelength, and $r_{0}$ is the slant range) is spatial frequency, $\eta_{i}=2 t_{i} / \lambda$ is temporal frequency, $\zeta_{i}=2 T_{i} / \lambda$ is thermal frequency with thermal baseline $T_{i}$, 
and $(\cdot)^{\mathrm{T}}$ is the transpose operation. The tomographic inversion can be written as follows (De Maio et al., 2009):

$\gamma(s, v, k)=\frac{\left|\boldsymbol{a}^{\mathrm{H}}(s, v, k) \boldsymbol{y}\right|}{\|\boldsymbol{a}(s, v, k)\|\|\boldsymbol{y}\|}$

where $(\cdot)^{\mathrm{H}}$ stands for the transpose and conjugate operation, $|\cdot|$ is the modulus of a complex number, and $\|\cdot\|$ is the Euclidean norm of a vector. Single and overlaid multiple scatterers can be detected by identifying the peaks of $\gamma$.

\section{Results and discussions}

Before performing the extended 4-D SAR, we need to confirm the presence of thermal expansion in phase data to avoid possible overfitting problems. To this end, we chose one pixel containing a single scatterer for time series analysis. The distribution of the residual phase after subtracting the height component and the distribution of the air temperature in the study site are depicted in Fig. 2. As expected, the phase is highly related to the temperature (correlation coefficient $=0.94$ ), and both have two seasonal periods in accordance with two years, indicating that thermal expansion is responsible for this nonlinear movement. Now we can reasonably exploit the extended 4-D SAR phase model to approximate the phase. In our experiment, single scatterers are detected by identifying the largest peaks in the reconstructed tomography.

\subsection{Validation of height estimates}

Fig. 3 (top) shows the height map estimated by the extended 4-D SAR. High-rise buildings are clearly visible in the height map. As B1 was still under construction at the beginning time of the acquisitions, the derived highest position is $345 \mathrm{~m}$ high, which is smaller than the final height (approximately $500 \mathrm{~m}$ ). The estimated height of B2 is $245 \mathrm{~m}$. To verify the height estimates of buildings, we compared the estimates with true height that was surveyed in 2011. Before comparison, we should geo-locate each persistent scatterer to the building that it belongs to. Automatic precise geo-location is difficult and out of the scope of this paper. Here, we use a simple method to geo-locate all the scatterers. We first geocoded the scatterers to the orthographic coordinate system using Gamma software and then used the known footprints of buildings to identify which building each scatterer belongs to. The highest scatterer in one footprint will be assumed to come from the roof of the building. The statistical results are depicted in Fig. 3 (bottom). Note that B1 is not included in the statistics because it was not completed in SAR estimates, but was completed when true height was acquired. The mean value and standard deviation of the differences between the true heights and the estimated heights are $1.9 \mathrm{~m}$ and $7.6 \mathrm{~m}$. We can see that the standard deviation is larger than the theoretical sub-meter accuracy in height estimation. This bias is primarily due to the geo-location error, that is to say, the two points for comparison may be not homologous. In our case, specifically, scatterers may be falsely geocoded to adjacent buildings due to the high density of buildings, or two points for comparison are unmatched because the roofs of buildings are often uneven. Notwithstanding the bias, the density plot of the scatters in Fig. 3 (bottom) indicates that the estimated height is in good agreement with the true height because most scatters gathers along the diagonal (the black line in Fig. 3 (bottom)). Of course, if we choose individual buildings manually for comparison, the accuracy is expected to be higher. The validity of height estimates can to some extent validate the estimated thermal amplitude and linearly deformation velocity because there is no particular reason why the estimation should be any better in one rather than in the other dimension (Ferretti et al., 2000).

\subsection{The effect of thermal expansion}

We present the thermal amplitude map (thermal map) in Fig. 4. We can see that the derived thermal amplitude is highly related to the building height. This relationship is most likely due to the accumulative effect of thermal expansion along the vertical direction (Crosetto et al., 2015). The fixed foundation and relaxed top result that when the temperature increases, dilation of each concrete member at different heights will accumulate upward, and thus the movement will approach the maximum value at the top of buildings in the upward direction, inducing movements toward the sensor in the radar line of sight (LOS) direction, whereas when the temperature decreases, concrete members will contract and the movement will approach the maximum value at the top of buildings in the downward direction, resulting in movements away from the sensor. The deformation amplitude associated with changes in temperature is therefore largest at the top of buildings and is reduced to zero at the bottom. In our case, the height of B1 ranges from 50 to $345 \mathrm{~m}$ as shown in Fig. 5 (left middle), and the thermal amplitude increases from $0.6 \mathrm{~mm} /{ }^{\circ} \mathrm{C}$ at $h=50 \mathrm{~m}$ to $2.3 \mathrm{~mm} /{ }^{\circ} \mathrm{C}$ at $h=345 \mathrm{~m}$ as shown in Fig. 5 (left top), whereas the derived height of B2 ranges from 5 to $245 \mathrm{~m}$ as shown in Fig. 5 (right middle), and $k$ increases from $0.1 \mathrm{~mm} /{ }^{\circ} \mathrm{C}$ at $h=5 \mathrm{~m}$ to $2.8 \mathrm{~mm} /{ }^{\circ} \mathrm{C}$ at $h=245 \mathrm{~m}$ as shown in Fig. 5 (right top). From Fig. 5
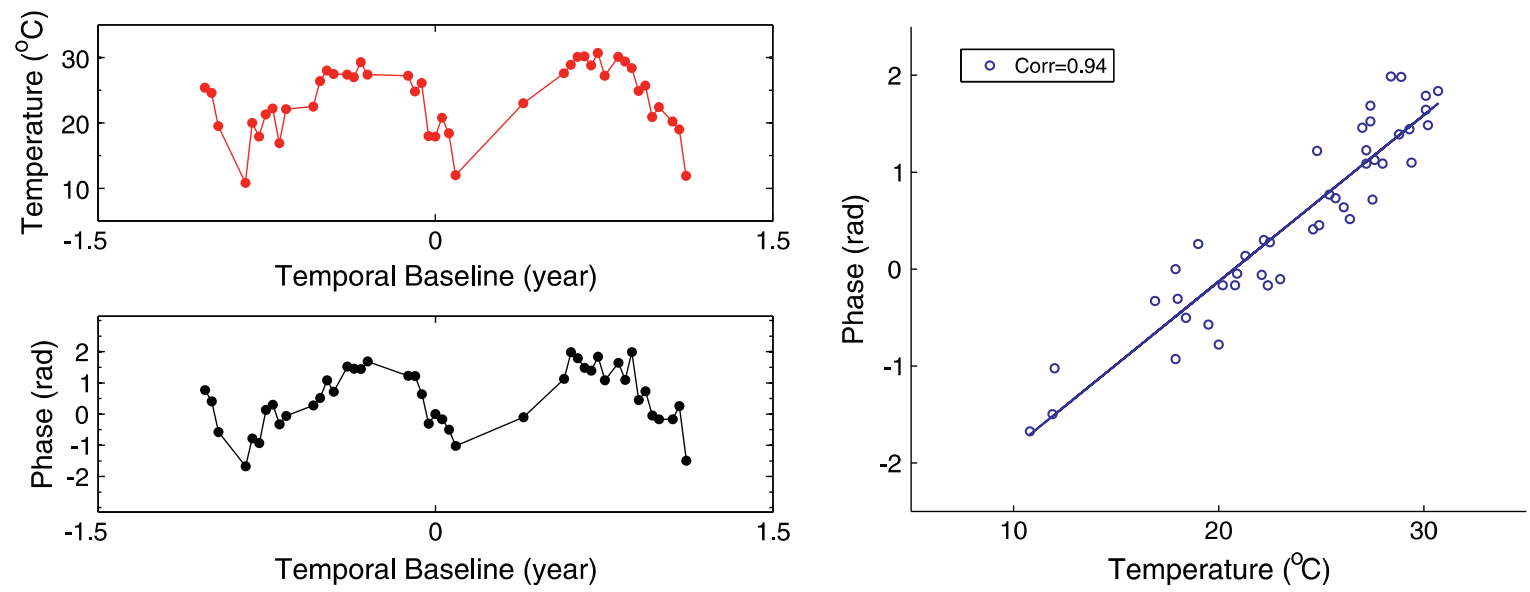

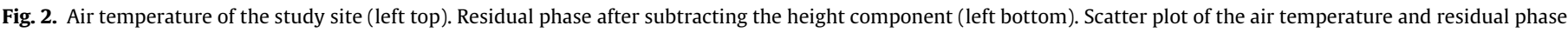
(right). 

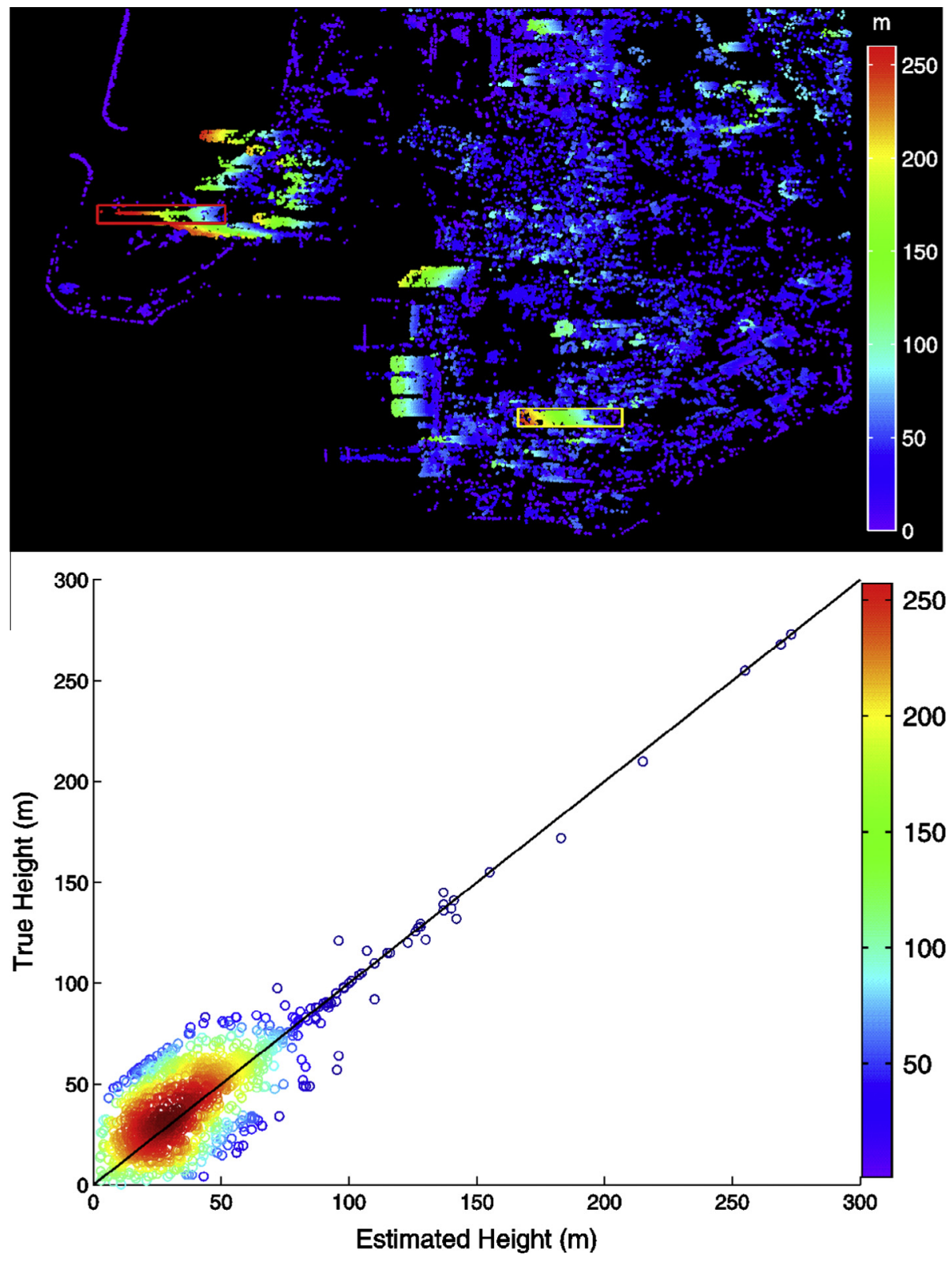

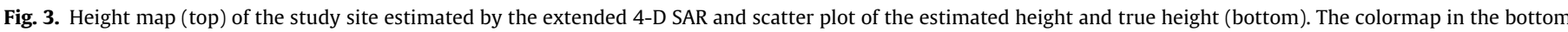

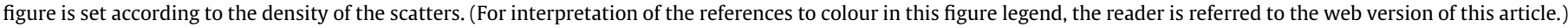

(left bottom and right bottom) we observe that the thermal amplitude is approximately proportional to the building height although there is some bias at the bottom and top parts of B2. The bias at the bottom part of B2 is most likely caused by mistakenly introducing surrounding structures that do not belong to B2 (Monserrat et al., 2011), and the bias at the top part of B2 is most likely caused by the phenomenon that the changes in temperature of the top part are larger than the changes in the air temperature that we used due to its direct exposure to sun. To facilitate analysis, we assume that the thermal amplitude is linearly related to the building height and then we can calculate the slope of the fitted regression line to be 0.0043 for B1 (Fig. 5 (left bottom)) and 0.0099 for B2 (Fig. 5 (right bottom)). That is to say, the gradient of thermal amplitude is $0.0043 \mathrm{~mm} /{ }^{\circ} \mathrm{C}$ per meter for $\mathrm{B} 1$ in the LOS direction, and 0 . $0099 \mathrm{~mm} /{ }^{\circ} \mathrm{C}$ per meter for B2. Assuming the thermal amplitude in the LOS direction is only caused by the upward accumulative effect of thermal expansion, we can then estimate the thermal expansion coefficient of $5.4 \times 10^{-6} /{ }^{\circ} \mathrm{C}$ for B1 and $12.4 \times 10^{-6} /{ }^{\circ} \mathrm{C}$ for B2. Note that $12.4 \times 10^{-6} /{ }^{\circ} \mathrm{C}$ is a typical value for concrete structures (Uygunoğlu and Topçu, 2009). There are two possible reasons for the estimated smaller thermal expansion coefficient of $\mathrm{B} 1$. The first reason is that the ratio of concrete mixes with the smaller thermal expansion coefficient in B1 is larger than that in $B 2$, reducing the thermal expansion coefficient of $B 1$, and the other one is that the changes in the building temperature of B1 are smaller than the changes in the air temperature that we used because the external glass screen window structure of B1 can resist temperature changes, decreasing the estimates of thermal amplitude.

\subsection{The effects of settlement, concrete creep and shrinkage}

The above thermal map demonstrates the movement associated with changes in temperature, and other movements, including settlement, creep and shrinkage, will be revealed in the linear deformation velocity map as shown in Fig. 6 (top). For comparison, we also present the linear deformation velocity map generated by 


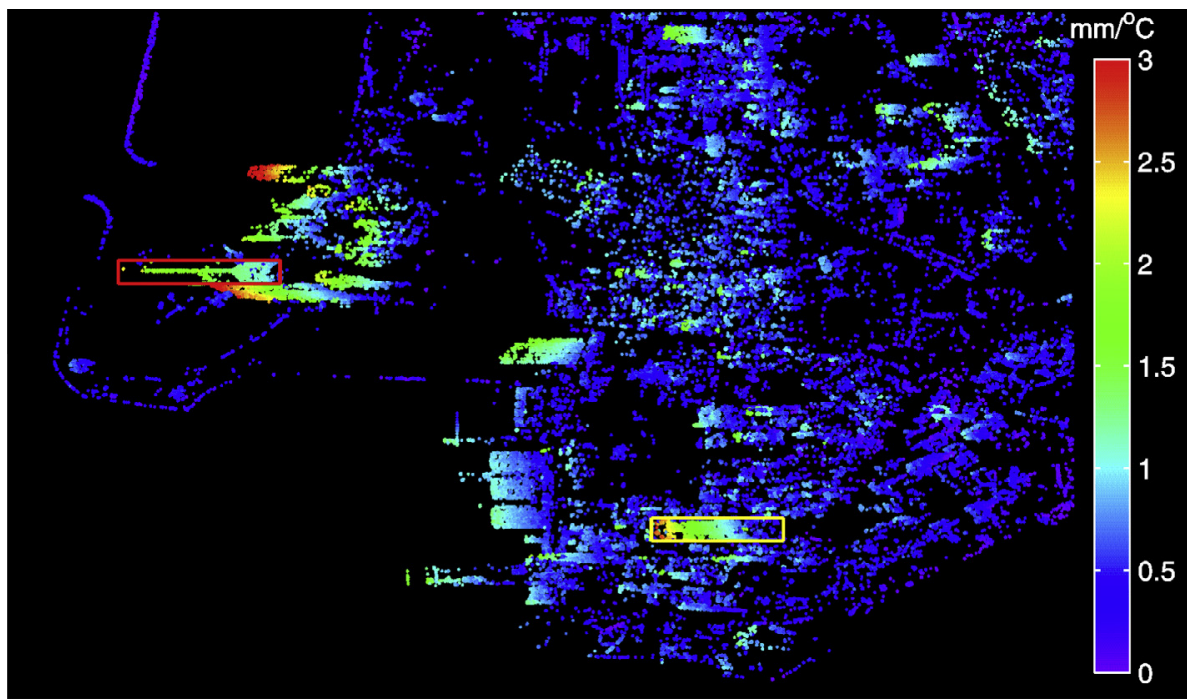

Fig. 4. Thermal map of the study site estimated by the extended 4-D SAR.
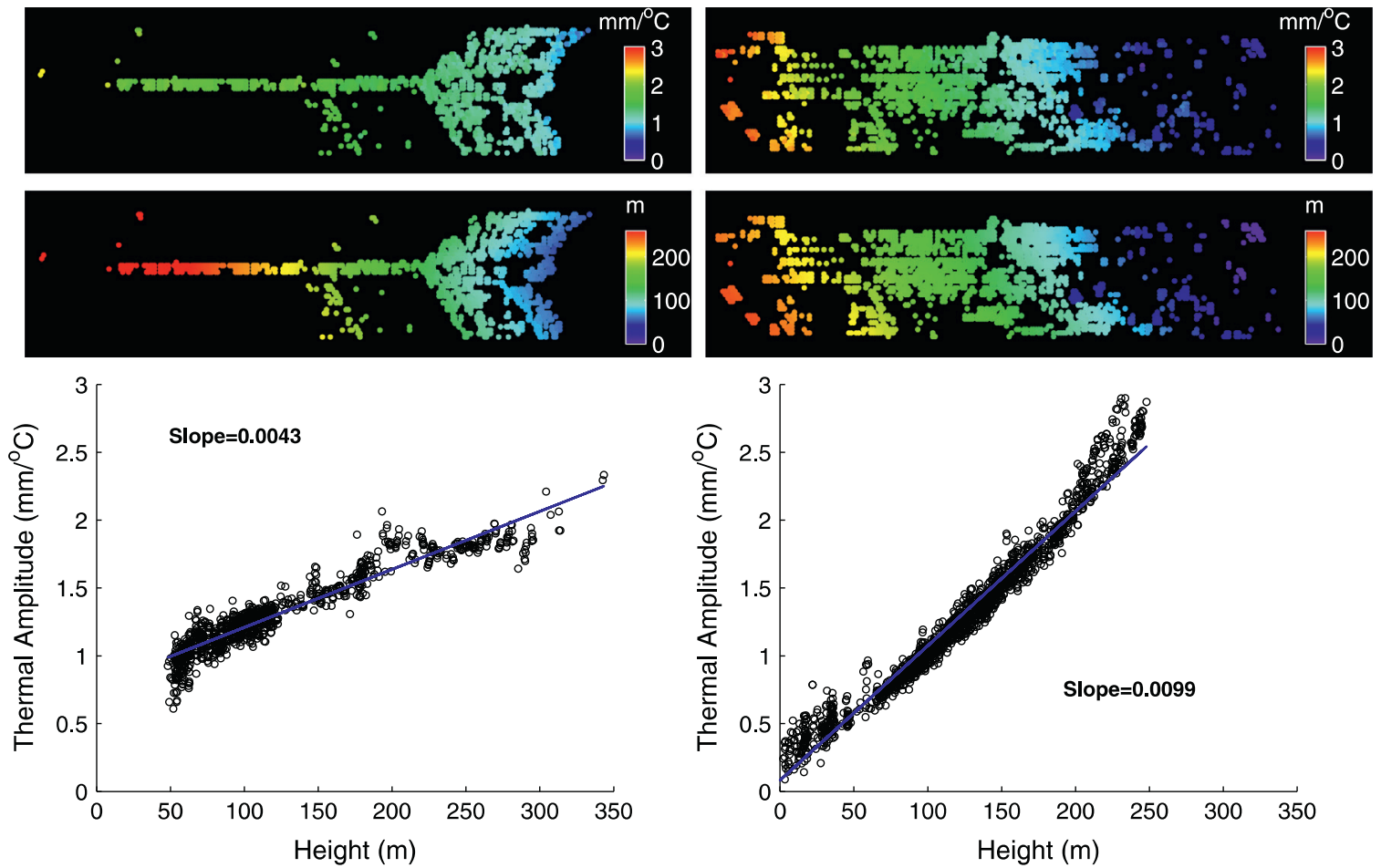

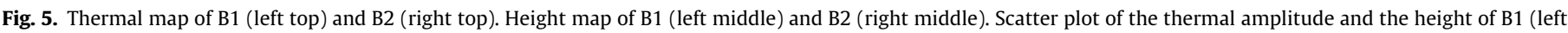
bottom) and B2 (right bottom).

4-D SAR in Fig. 6 (bottom). The linear deformation velocity by 4-D SAR can be biased in the presence of thermal expansion due to the correlation between the temporal frequency and the thermal frequency. We calculated the correlation coefficient to be 0.15 in our case. By comparing the deformation estimates by 4-D SAR and the extended 4-D SAR, we obtain that 4-D SAR can result in a bias of $2-3 \mathrm{~mm} / \mathrm{y}$ in the estimated linear deformation velocity when $1 \mathrm{~mm} /{ }^{\circ} \mathrm{C}$ thermal amplitude is present. The linear deformation velocity of old buildings by 4-D SAR is therefore height-dependent, such as the building in the white rectangle in Fig. 6 (bottom), whereas this height-dependent pattern disappears when using the extended 4-D SAR phase model as shown in Fig. 6 (top). We can therefore infer that the thermal expansion component is properly removed or at least is negligible in the deformation velocity map by the extend 4-D SAR. From Fig. 6 (top) we can see that the old buildings become stable after eliminating the influence of thermal expansion, whereas the two newly built buildings B1 and B2 still exhibit height-dependent dynamic behaviors.

First, from the linear velocity map of B1 (Fig. 7 (left top)), we can observe that the velocity (approximately $-3 \mathrm{~mm} / \mathrm{y}$ ) at $h=50 \mathrm{~m}$ is much smaller than the velocity (approximately $1 \mathrm{~mm} / \mathrm{y}$ ) of the surrounding ground surface, indicating that B1 is subject to settlement during this period, causing the tower to move away from the sensor in the LOS direction. The settlement of B1 has been confirmed by the construction company. This settlement is the 

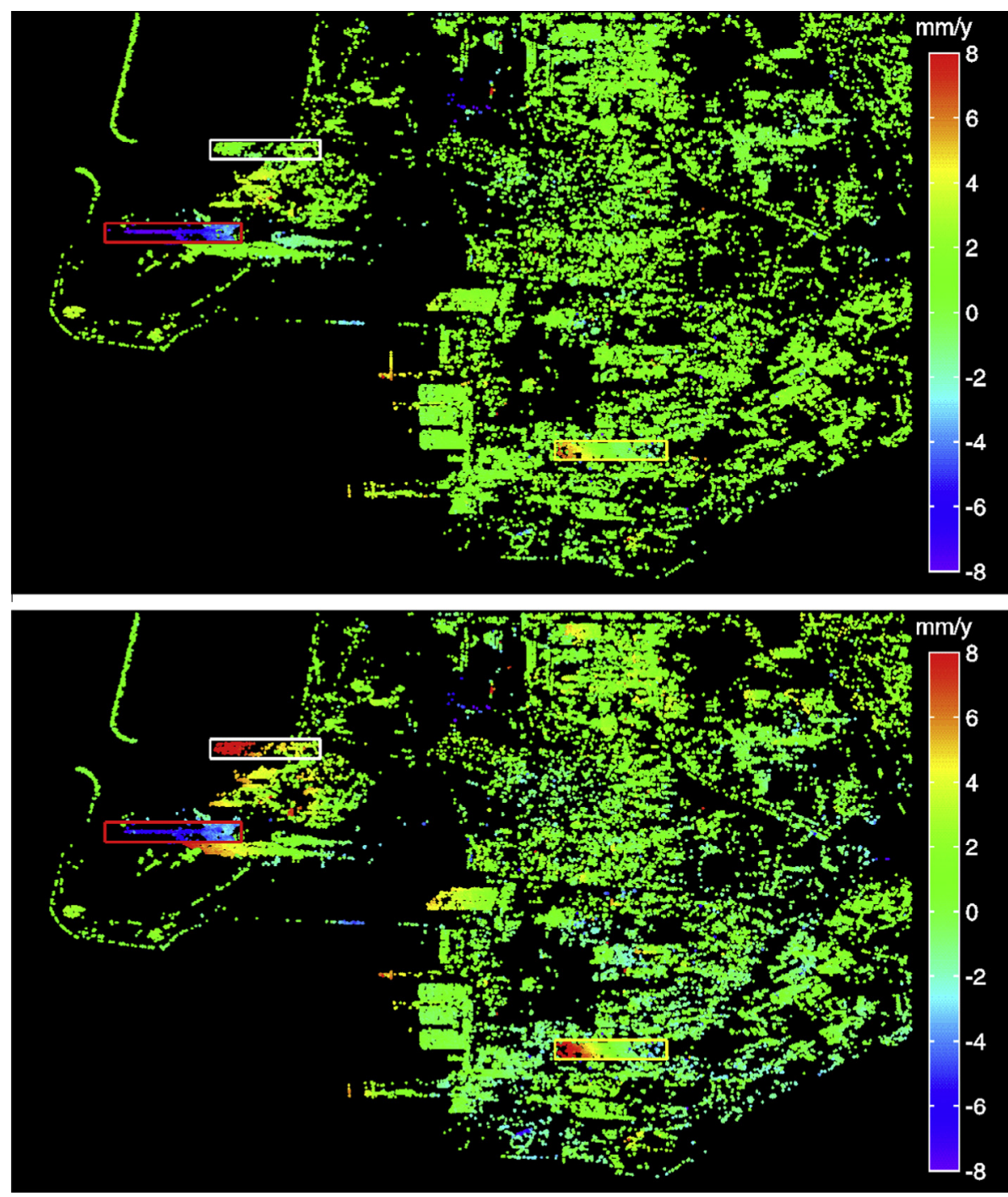

Fig. 6. Linear deformation velocity map estimated by the extended 4-D SAR (top) and 4-D SAR (bottom).
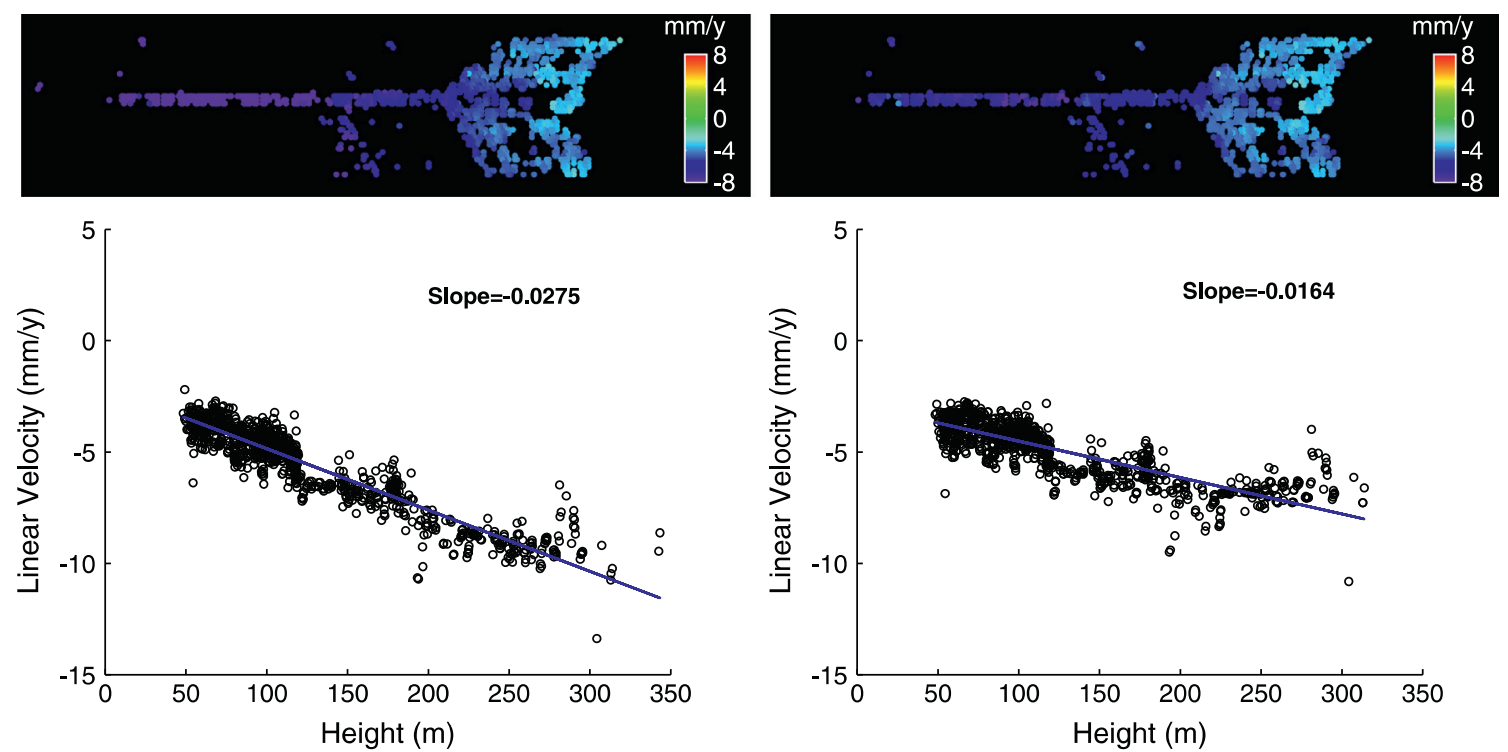

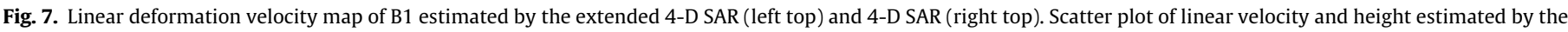
extended 4-D SAR (left bottom) and 4-D SAR (right bottom). 
combined result of the high sustained load of this superstructure and the consolidation process of the designed raft foundation. It should be noted that the settlement is a height-independent movement in the sense that structural components at different heights have the same linear velocity that theoretically equals to the velocity of the bottom. In addition to the settlement, we also notice a height-dependent movement that the linear velocity is $-3 \mathrm{~mm} / \mathrm{y}$ at $h=50 \mathrm{~m}$ and decreases to $-9.5 \mathrm{~mm} / \mathrm{y}$ at $h=345 \mathrm{~m}$; that is to say, structural components at higher positions move away from the sensor more quickly than those at lower positions. This height-dependent pattern has been confirmed to be caused by wall shortening under the combined effects of concrete creep and shrinkage. For one thing, the high load of B1 due to the building weight leads to downward creep that shortens the wall. For another, the natural shrinkage of concrete members possibly caused by the loss of water also cause a significant volume contraction of the wall. The synthetic effects induce height-dependent downward movements. As creep and shrinkage both cause downward movements, they are difficult to separate from the linear velocity map. If we aim to separate creep and shrinkage, more structural knowledge and predication models are required. The deformed shape of B1 is illustrated in Fig. 8 (left). Assuming that the settlement is $\Delta 2$ and that the deformation of the top position is $\Delta 1, \Delta 1$ is larger than $\Delta 2$ due to wall shortening. From Fig. 7 (left bottom), we calculate that the gradient of linear velocity due to wall shortening is $-0.0275 \mathrm{~mm} / \mathrm{y}$ per meter. Then, we can infer the linear deformation velocity of the bottom floor $(h=0 \mathrm{~m})$ to be approximately $-1.6 \mathrm{~mm} / \mathrm{y}$ using the velocity at $h=50 \mathrm{~m}$. Consequently, the velocity difference between the bottom floor and the surrounding ground surface is $2.6 \mathrm{~mm} / \mathrm{y}$ in the LOS direction, and the vertical settlement can be calculated to be $3.27 \mathrm{~mm} / \mathrm{y}$ after being divided by $\cos (\theta)$ ( $\theta$ is the incidence angle) if we assume the surrounding ground surface is stable. This settlement is smaller than the limit of $4 \mathrm{~mm} / \mathrm{y}$ as described above and is therefore acceptable. From Fig. 7 we can see that the slope $(-0.0164)$ of the fitted regression line from the result by 4-D SAR is larger than that $(-0.0275)$ by the extended 4 -D SAR; that is to say, the absolute linear velocity difference by the 4-D SAR is smaller than that by the extended 4-D SAR at the same height difference. This is because the velocity by 4-D SAR involves the contribution of thermal expansion; more precisely, the upward accumulative effect of thermal expansion counteracts a portion of downward deformation of wall shortening. As a consequence, 4-D SAR leads to the underestimation of concrete creep and shrinkage and thus if we expect to extract precise creep and shrinkage it is necessary to get rid of thermal expansion beforehand. To study the evolution of creep and shrinkage, we retrieved the time series deformation of one point on the wall with reference to one lower point on the wall as shown in Fig. 8 (right top). The height difference of the two points is $40 \mathrm{~m}$. There is a distinct seasonal trend associated with changes in temperature in time series. After removing thermal expansion and low-pass filtering, the residual deformation is presented in Fig. 8 (right bottom). The fact that the residual deformation has no seasonal trends can also indicate that the thermal expansion has been properly removed by the extended 4-D SAR. The residual deformation represents the relative movements of the two points induced by wall shortening, from which we can see that the rate of creep and shrinkage is almost steady and does not decline during this period.

Unlike B1, the building B2 shows an opposite dynamic behavior (Fig. 9 (left top)) that structural components at higher positions move toward the sensor more quickly than the components at lower positions. This phenomenon has been confirmed to be caused by wall deflection due to asymmetrical creep induced differential wall shortening. Fig. 10 (left) illustrates the deformed shape of B2. This building involves a podium structure (approximately $45 \mathrm{~m}$ high) and an upper structure (approximately $200 \mathrm{~m}$ high). The weight of the left part of the upper structure is designed to be larger than that of the right part so that it sustains higher stress than the right part, which causes differential wall shortening that incurs deflection of the upper structure toward the left as shown in Fig. 9 (left). The deformation of wall deflection is largest at the top of the tower and will be reduced to zero at the bottom. As a result, there are two height-dependent movements (wall shortening and wall deflection) in B2. It should be noted that if the building inclines oppositely or the sensor illuminates the building from the east (descending mode), wall deflection will be merged with wall shortening because they both cause movements away from the sensor. Assuming that wall deflection only induces horizontal movements and wall shortening only induces vertical movements, the directions of the two movements are opposite when projected to the LOS direction. The deformation components
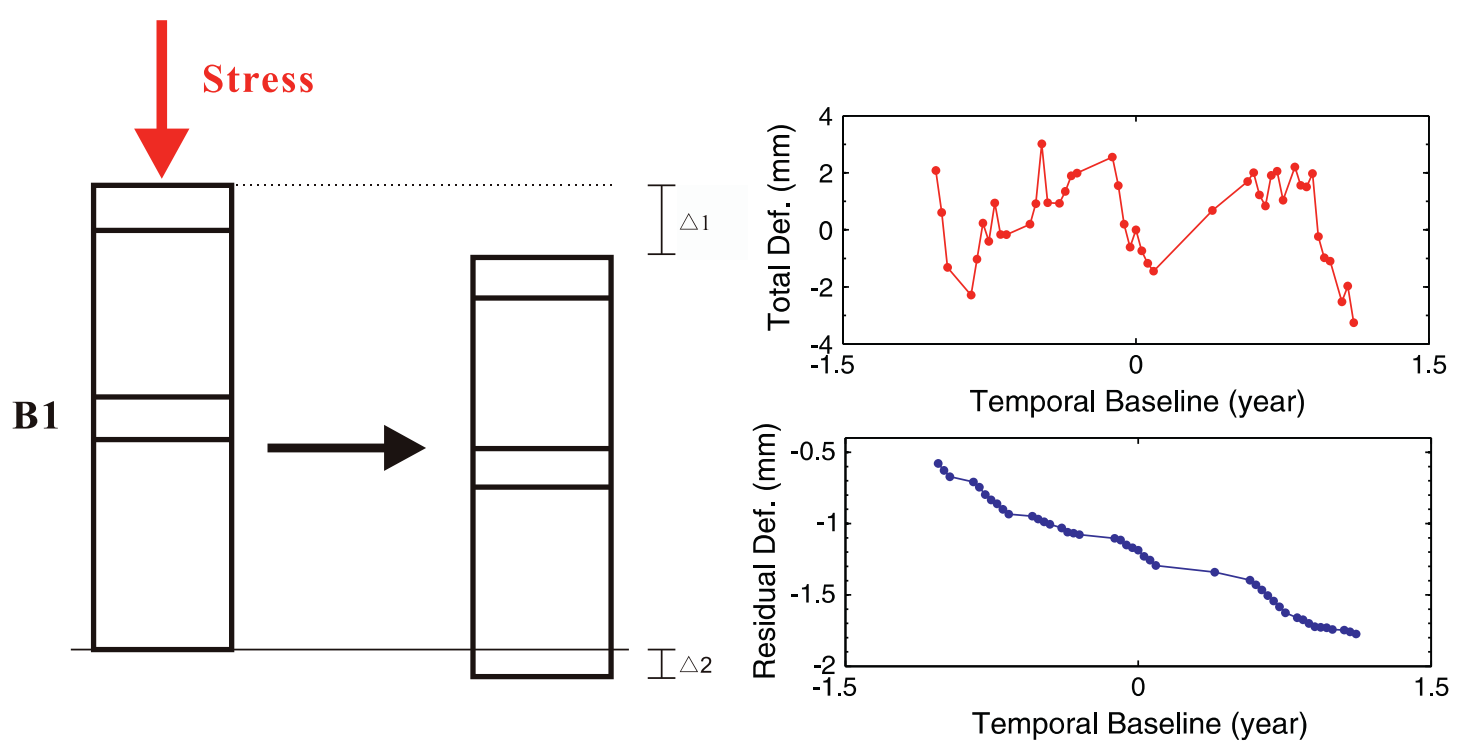

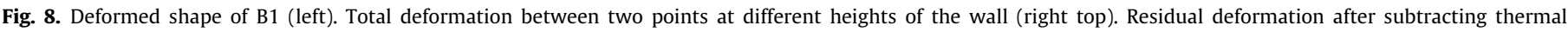
expansion and low-pass filtering (right bottom). 

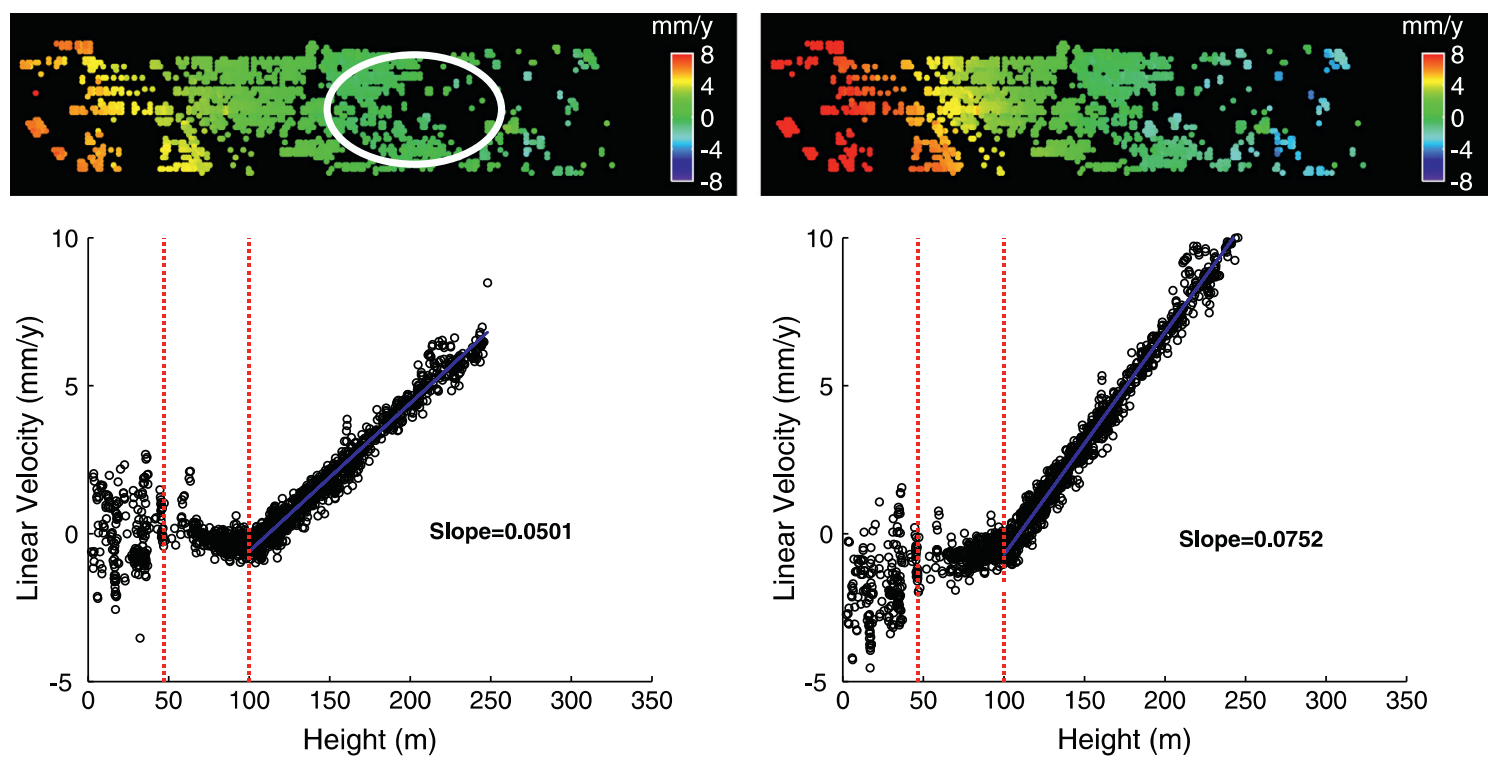

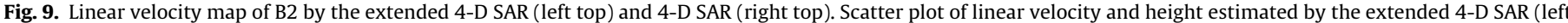
bottom) and 4-D SAR (right bottom).
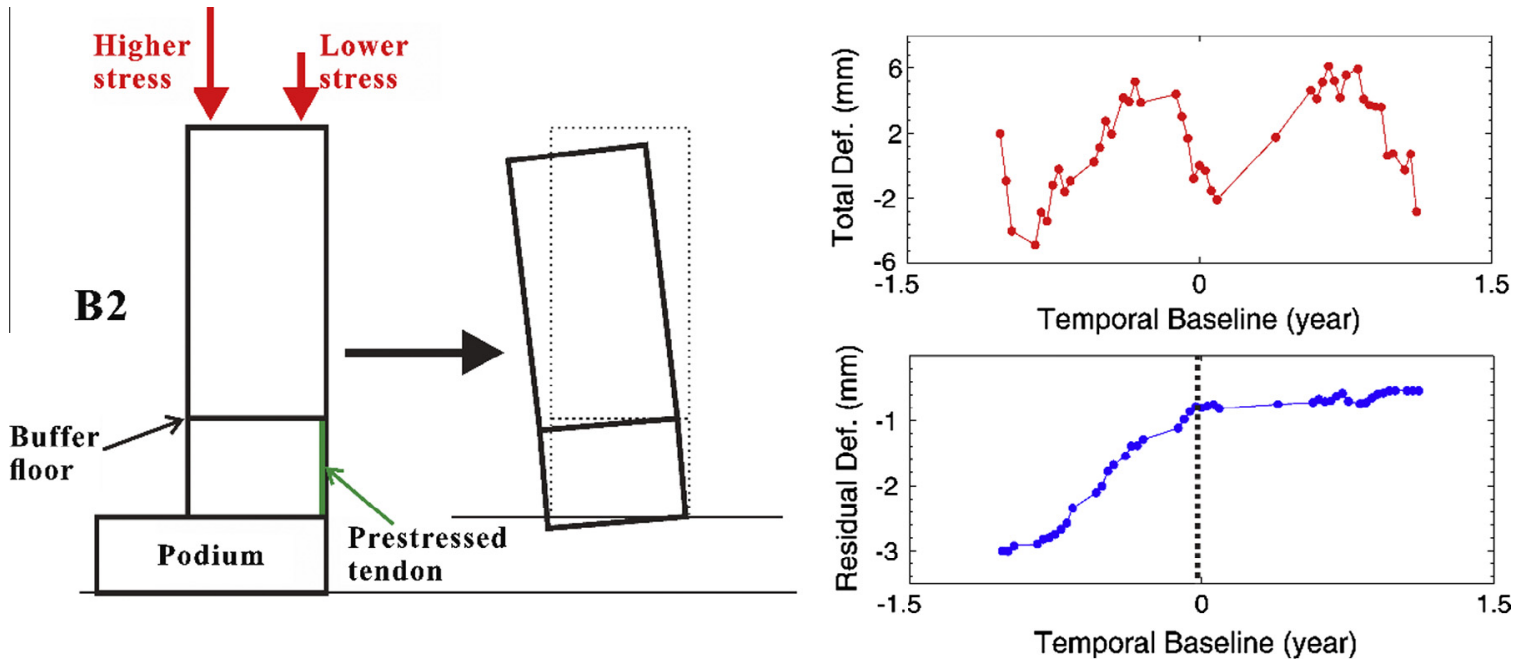

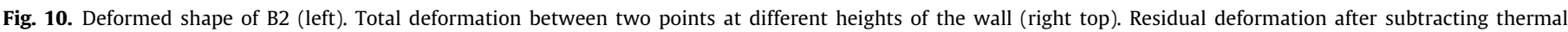
expansion and low-pass filtering (right bottom).

of wall deflection and wall shortening in the LOS direction are therefore counteractive and the linear velocity map in Fig. 9 (left top) only measures their combined results. We observe that the velocity (approximately $-1 \mathrm{~mm} / \mathrm{y}$ ) of the lower positions (the white circle in Fig. 9 (left top)) of B2 are smaller than the velocity $(1 \mathrm{~mm} / \mathrm{y}$ ) of the surrounding ground surface, which is because the deformation component of wall shortening in the LOS direction exceeds the deformation component of wall deflection in the LOS direction. With the increase of height, the deformation component of wall deflection overwhelms the deformation component of wall shortening, and therefore, the top positions of the building moves toward the sensor. Fig. 9 (left bottom) gives the scatter plot of linear velocity and height of B2 by the extended 4-D SAR. As the podium structure that is lower than $45 \mathrm{~m}$ (the left red dotted line ${ }^{1}$

\footnotetext{
${ }^{1}$ For interpretation of color in Fig. 9, the reader is referred to the web version of this article.
}

in Fig. 9 (left bottom)) is only subject to wall shortening, the velocity should decline with the increasing height. As described above, the statistical results at lower positions may falsely include some surrounding structures that do not belong to the building, and thus the relationship appears to be distorted. The upper structure ranges from $45 \mathrm{~m}$ to $245 \mathrm{~m}$, and we can divide the upper structure into two parts: the lower part ranging from $45 \mathrm{~m}$ to $100 \mathrm{~m}$ (the right red dotted line in Fig. 9 (left bottom)), and the higher part ranging from $100 \mathrm{~m}$ to $245 \mathrm{~m}$. At the lower part, linear velocity slightly declines with increasing height, indicating that the gradient of linear velocity due to wall shortening is larger than that due to wall deflection, whereas in the higher part, linear velocity sharply increases with the increasing height, indicating the gradient of linear velocity due to wall deflection exceeds that due to wall shortening. From the structural layout of B2 in Fig. 10 (left), we can see that there is a buffer floor that is located at approximately $100 \mathrm{~m}$ high, and a prestressed tendon is designed at the lower part. As a consequence, 
the difference of the gradient between the lower part and the higher part is most likely caused by the use of the prestressed tendon to resist possible excessive deflection. In the case of the higher part, if we assume that the linear velocity is linearly related to the height, we can obtain that the gradient of linear velocity is $0.0501 \mathrm{~mm} / \mathrm{y}$ per meter in the LOS direction. It should be noted that we cannot derive horizontal movements resulting from wall deflection and vertical movements resulting from wall shortening using single track images. To achieve this, both the ascending and descending images are required. According to (Eurocode-EN 1990, 2002) the deflection limit is $h / 500$ for the entire building, i.e., $400 \mathrm{~mm}$ for B2 with $200 \mathrm{~m}$ high. The deformation performance of B2 seems to be satisfactory according to our measurements. From Fig. 9 we observe that the gradient of absolute linear velocity by 4-D SAR is larger than that by the extended 4-D SAR at the same height difference. This result is also attributed to the upward accumulative effect of thermal expansion; in other words, the deformation component of thermal expansion has the same direction with the deformation component of wall deflection in the LOS direction, which leads to the overestimation of wall deflection. In fact, if we do not remove thermal expansion, the deflection cannot be discovered because the deformation velocities of all buildings will exhibit height-dependent trends in the linear deformation velocity map as shown in Fig. 6 (bottom). It should also be noted that if the building inclines oppositely or the sensor illuminates the building from the east (descending mode), the presence of thermal expansion will lead to the underestimation of wall deflection because their deformation components in the LOS direction will be counteractive.

As deflection due to creep is time-dependent, we are more concerned with the evolution of deflection than the magnitude of deflection. Fig. 10 (right top) presents the time series deformation of two points on the wall with a height difference of $50 \mathrm{~m}$. Two seasonal periods are also evident in the total deformation. After removing thermal expansion and low-passing filtering, we obtained the residual deformation as shown in Fig. 10 (right bottom). Before November 2009 (the black dotted line) there is a continuous relative movement between the two points but the movement slows down gradually, indicating that deflection is continuous but its rate keeps declining, after November 2009 the trend becomes nearly stationary, indicating that the deflection becomes very slow at that time and that the deformation components of wall deflection and wall shortening in the LOS direction are almost equivalent.

\section{Conclusion}

The dynamic behavior of buildings at early ages can significantly influence the serviceability of buildings and should be constantly inspected carefully. This paper discusses the possibility and potential of multi-dimensional SAR tomography for monitoring the deformation of newly built concrete buildings, including thermal expansion, settlement, concrete creep and shrinkage. The extended 4-D SAR is exploited to separate thermal expansion from total deformations. Thermal expansion is illustrated in the thermal map, and settlement combined with creep and shrinkage is revealed in the linear deformation velocity map. A special focus is on the elaboration of wall shortening and wall deflection caused by creep and shrinkage. For comparison, the estimates by 4-D SAR are also presented. Several conclusions can be summarized as follows:

(1) Normally, the slow deformation of newly built concrete buildings that can be captured by SAR consists of thermal expansion, possible settlement, creep and shrinkage. These movements can be separately identified by the extended
4-D SAR. Thermal expansion is demonstrated in the thermal map. Settlement together with concrete creep and shrinkage can be quantified in the linear deformation velocity map.

(2) Thermal expansion repeats periodically with seasonal changes in temperature throughout the entire lifetime of the buildings. This expansion can cause the high-rise building to dilate and contract periodically in the vertical direction due to the upward accumulative effect. In addition, different buildings may have different thermal expansion levels (B1 and B2 in our case), which is likely attributed to the different thermal expansion coefficients of the construction materials or mismatching of the employed temperature.

(3) Creep and shrinkage are two long-term movements that only occur in concrete structures. Normally, their rates are largest at the outset and gradually decline as the load is relaxed and dehydration proceeds. Creep and shrinkage can lead to wall shortening (B1 in our case). Moreover, asymmetrical creep can trigger wall deflection (B2 in our case). Wall shortening and wall deflection are both height-dependent movements in the linear velocity map. Specifically, wall shortening induces movements that increase with the increasing height in the downward direction. Wall deflection induces movements that increase with the increasing height in the deflection direction; that is to say, the deformation component is toward the sensor in the LOS direction if the building inclines toward the sensor (B2 in our case) and is away from the sensor if the building inclines oppositely. In addition, only if the building inclines toward the sensor (B2 in our case), the deflection can be discovered from the linear deformation velocity map; otherwise, the deflection will be merged with wall shortening because the direction of its deformation component is same with that of wall shortening.

(4) To measure wall shortening and wall deflection due to creep and shrinkage, it is necessary to get rid of the influence of thermal expansion; otherwise, it may conceal possible wall shortening and wall deflection, and bias the deformation estimates. Specifically, the upward accumulative effect of thermal expansion counteracts the downward movements due to wall shortening, resulting in the underestimation of wall shortening. In the case of wall deflection, if the building inclines toward the sensor, thermal expansion can lead to the overestimation of wall deflection (B2 in our case), whereas if the building inclines oppositely, thermal expansion can lead to the underestimation of wall deflection.

(5) The contributions of creep and shrinkage are commonly difficult to separate from the linear deformation velocity map because both of them yield downward movements. To achieve this, interdisciplinary knowledge, especially the structural knowledge of buildings and relevant predication models, should be known preliminarily. In addition, to extract the vertical movements resulting from wall shortening and the horizontal movements resulting from wall deflection, both ascending and descending images are required.

In short, the technique provides a promising tool for the constant inspection of newly built concrete buildings. Future study will focus on improving the robustness of the technique and applying the technique to assist monitoring more newly built concrete buildings with different structural designs.

\section{Acknowledgements}

This work was supported in part by National Key Basic Research Program of China under Grant 2015CB954103, in part by State Key 
Laboratory of Resources and Environmental Information System, in part by the Innovation and Technology Support Programme of HKSAR, China under Grant ITS/075/13, and in part by the Innovation and Technology Support Programme of HKSAR, China under Grant UIM/264. We would like to thank Dr. Yong Xu for his help in validation of height estimates. We also thank the anonymous reviewers for their comments and corrections.

\section{References}

Bazant, Z.P., Wittmann, F.H., 1982. Creep and Shrinkage in Concrete Structures. Wiley, New York.

Budillon, A., Evangelista, A., Schirinzi, G., 2011. Three-dimensional SAR focusing from multipass signals using compressive sampling. Geosci. Remote Sens., IEEE Trans. 49 (1), 488-499.

Colesanti, C., Ferretti, A., Novali, F., Prati, C., Rocca, F., 2003. SAR monitoring of progressive and seasonal ground deformation using the permanent scatterers technique. Geosci. Remote Sens., IEEE Trans. 41 (7), 1685-1701.

Crosetto, M., Monserrat, O., Cuevas-González, M., Devanthéry, N., Luzi, G., Crippa, B. 2015. Measuring thermal expansion using X-band persistent scatterer interferometry. ISPRS J. Photogrammetry Remote Sens. 100, 84-91.

De Maio, A., Fornaro, G., Pauciullo, A., 2009. Detection of single scatterers in multidimensional SAR imaging. Geosci. Remote Sens., IEEE Trans. 47 (7), 2284 2297.

Ferretti, A., Prati, C., Rocca, F., 2000. Nonlinear subsidence rate estimation using permanent scatterers in differential SAR interferometry. Geosci. Remote Sens., IEEE Trans. 38 (5), 2202-2212.

Fornaro, G., Serafino, F., Soldovieri, F., 2003. Three-dimensional focusing with multipass SAR data. Geosci. Remote Sens., IEEE Trans. 41 (3), 507-517.

Fornaro, G., Reale, D., Serafino, F., 2009. Four-dimensional SAR imaging for height estimation and monitoring of single and double scatterers. Geosci. Remote Sens., IEEE Trans. 47 (1), 224-237.

Fornaro, G., Reale, D., Verde, S., 2013. Bridge thermal dilation monitoring with millimeter sensitivity via multidimensional SAR imaging. Geosci. Remote Sens. Lett., IEEE 10 (4), 677-681.
Gernhardt, S., Bamler, R., 2012. Deformation monitoring of single buildings using meter-resolution SAR data in PSI. ISPRS J. Photogrammetry Remote Sens. 73, 68-79.

Gernhardt, S., Adam, N., Eineder, M., Bamler, R., 2010. Potential of very high resolution SAR for persistent scatterer interferometry in urban areas. Ann. GIS 16 (2), 103-111.

Gilbert, R., 2001. Shrinkage, cracking and deflection-the serviceability of concrete structures. Electron. J. Struct. Eng. 1 (1), 2-14.

Lombardini, F., 2005. Differential tomography: a new framework for SAR interferometry. Geosci. Remote Sens., IEEE Trans. 43 (1), 37-44.

Ma, P., Lin, H., Lan, H., Chen, F., 2014. On the performance of reweighted L1 minimization for tomographic SAR imaging. Geosci. Remote Sens. Lett. IEEE (99), 1-5.

Monserrat, O., Crosetto, M., Cuevas, M., Crippa, B., 2011. The thermal expansion component of persistent scatterer interferometry observations. Geosci. Remote Sens. Lett., IEEE 8 (5), 864-868.

Reale, D., Fornaro, G., Pauciullo, A., Zhu, X., Bamler, R., 2011. Tomographic imaging and monitoring of buildings with very high resolution SAR data. Geosci. Remote Sens. Lett., IEEE 8 (4), 661-665.

Reale, D., Fornaro, G., Pauciullo, A., 2013. Extension of 4-D SAR imaging to the monitoring of thermally dilating scatterers. Geosci. Remote Sens. IEEE Trans. (99), 1-11

Reigber, A., Moreira, A., 2000. First demonstration of airborne SAR tomography using multibaseline L-band data. Geosci. Remote Sens., IEEE Trans. 38 (5), 2142 2152.

Stoica, P., Moses, Randolph L., 2005. Spectral Analysis of Signals. Prentice-Hall Englewood Cliffs, NJ.

Uygunoğlu, T., Topçu, İ.B., 2009. Thermal expansion of self-consolidating norma and lightweight aggregate concrete at elevated temperature. Constr. Build. Mater. 23 (9), 3063-3069.

Van Waning, H., 2014. A Feasibility Study of Building Monitoring and Forensic Engineering with Interferometric Synthetic Aperture Radar. Delft University of Technology, TU Delft.

Zhu, X.X., Bamler, R., 2010. Very high resolution spaceborne SAR tomography in urban environment. Geosci. Remote Sens., IEEE Trans. 48 (12), 4296-4308.

Zhu, X.X., Bamler, R., 2011. Let's do the time warp: multicomponent nonlinear motion estimation in differential SAR tomography. Geosci. Remote Sens. Lett. IEEE 8 (4), 735-739. 